\title{
Failure of Acute Ethanol Administration to Alter Cerebrocortical and Hippocampal Allopregnanolone Levels in C57BL/6J and DBA/2J Mice
}

\author{
Patrizia Porcu, Andrea Locci, Francesca Santoru, Roberta Berretti, A. Leslie Morrow, and \\ Alessandra Concas
}

\begin{abstract}
Background: Ethanol (EtOH) administration increases brain allopregnanolone levels in rats, and this increase contributes to sensitivity to EtOH's behavioral effects. However, EtOH's effects on allopregnanolone may differ across species. We investigated the effects of acute EtOH administration on allopregnanolone, progesterone, and corticosterone levels in cerebral cortex and hippocampus of $\mathrm{C} 57 \mathrm{BL} / 6 \mathrm{~J}$ and $\mathrm{DBA} / 2 \mathrm{~J}$ mice, 2 inbred strains with different alcohol sensitivity.

Methods: Naive male C57BL/6J and DBA/2J mice received EtOH $(1,2,3$, or $4 \mathrm{~g} / \mathrm{kg}$, intraperitoneally [i.p.]) or saline and were euthanized 1 hour later. For the time-course study, mice received EtOH ( $2 \mathrm{~g} / \mathrm{kg}$, i.p.) and were euthanized 15, 30, 60, and 120 minutes later. Steroids were measured by radioimmunoassay.

Results: Acute EtOH administration did not alter cerebrocortical and hippocampal levels of allopregnanolone and progesterone in these strains at any of the doses and time points examined. Acute EtOH dose-dependently increased cerebrocortical corticosterone levels by 319, 347, and 459\% in $\mathrm{C} 57 \mathrm{BL} / 6 \mathrm{~J}$ mice at the doses of 2,3 , and $4 \mathrm{~g} / \mathrm{kg}$, and by $371,507,533$, and $692 \%$ in DBA $/ 2 \mathrm{~J}$ mice at the doses of 1, 2, 3, and $4 \mathrm{~g} / \mathrm{kg}$, respectively. Similar changes were observed in the hippocampus. EtOH's effects on cerebrocortical corticosterone levels were also time dependent in both strains. Moreover, acute EtOH administration time-dependently increased plasma levels of progesterone and corticosterone. Finally, morphine administration increased cerebrocortical allopregnanolone levels in C57BL/6J $(+77,+93$, and $+88 \%$ at 5,10 , and $30 \mathrm{mg} / \mathrm{kg}$, respectively) and DBA $/ 2 \mathrm{~J}$ mice $(+81 \%$ at $5 \mathrm{mg} / \mathrm{kg})$, suggesting that the impairment in brain neurosteroidogenesis may be specific to EtOH.

Conclusions: These results underline important species differences on EtOH-induced brain neurosteroidogenesis. Acute EtOH increases brain and plasma corticosterone levels but does not alter cerebrocortical and hippocampal concentrations of allopregnanolone and progesterone in naïve $\mathrm{C} 57 \mathrm{BL} / 6 \mathrm{~J}$ and DBA/2J mice.
\end{abstract} Mice.

Key Words: Allopregnanolone, Progesterone, Corticosterone, Ethanol, C57BL/6J and DBA/2J

\begin{abstract}
A LLOPREGNANOLONE, THE $3 \alpha, 5 \alpha$-reduced metabolite of progesterone, is a neuroactive steroid that produces inhibitory neurobehavioral effects such as anxiolytic, anticonvulsant, and sedative/hypnotic actions via interaction with $\gamma$-aminobutyric acid type $\mathrm{A}\left(\mathrm{GABA}_{\mathrm{A}}\right)$ receptors (Biggio and Purdy, 2001). Allopregnanolone concentrations are altered in several neuropsychiatric disorders, including anxiety, depression, premenstrual dysphoric disorder,

From the Institute of Neuroscience (PP, AC), National Research Council of Italy (CNR), Cagliari, Italy; Department of Life and Environment Sciences ( $A L, F S, R B, A C$ ), Section of Neuroscience, University of Cagliari, Cagliari, Italy; and Departments of Psychiatry and Pharmacology (ALM), Bowles Center for Alcohol Studies, University of North Carolina School of Medicine, Chapel Hill, North Carolina.

Received for publication July 19, 2013; accepted October 25, 2013.

Reprint requests: Patrizia Porcu, PhD, Institute of Neuroscience, National Research Council of Italy (CNR), Cittadella Universitaria, 09042 Monserrato, Cagliari, Italy; Tel.: + 39070675 4163; Fax: +39070675 4166; E-mail:patrizia.porcu@in.cnr.it

Copyright $₫ 2014$ by the Research Society on Alcoholism.
\end{abstract}

DOI: $10.1111 /$ acer.12329 schizophrenia, and drug addiction (Biggio and Purdy, 2001; Morrow et al., 2006). Allopregnanolone levels are increased in rat brain and plasma by administration of various psychoactive drugs, including ethanol (EtOH; Morrow et al., 1999), caffeine (Concas et al., 2000), nicotine (Porcu et al., 2003), tetrahydrocannabinol (Grobin et al., 2005), morphine (Concas et al., 2006; Grobin et al., 2005), and gammahydroxybutyric acid (Porcu et al., 2004).

Systemic EtOH administration increases brain and plasma allopregnanolone levels to physiologically relevant concentrations, capable of enhancing GABAergic transmission and contribute to sensitivity to behavioral effects of EtOH in rats (Barbaccia et al., 1999; Morrow et al., 1999; VanDoren et al., 2000). In fact, allopregnanolone modulates EtOH's anticonvulsant effects (VanDoren et al., 2000), sedation (Khisti et al., 2003), impairment of spatial memory (Matthews et al., 2002), anxiolytic-like (Hirani et al., 2005), antidepressant-like (Hirani et al., 2002), and proaggressive actions (Fish et al., 2001). Allopregnanolone also mediates EtOH's ability to inhibit the spontaneous firing of medial septal neurons (VanDoren et al., 2000) and hippocampal 
pyramidal neurons (Tokunaga et al., 2003) in rats. These behavioral and electrophysiological responses are inhibited by pretreatment with finasteride (a $5 \alpha$-reductase inhibitor) and/or by prior adrenalectomy. In fact, the EtOH-induced increase in cerebrocortical allopregnanolone levels is mediated by the hypothalamic-pituitary-adrenal (HPA) axis, since it is absent following adrenalectomy/gonadectomy (Khisti et al., 2003; O'Dell et al., 2004; Porcu et al., 2004) or hypophysectomy (Boyd et al., 2010). However, EtOH can increase allopregnanolone content in hippocampal slices from intact and adrenalectomized/gonadectomized rats (Follesa et al., 2006).

The evidence regarding a role for allopregnanolone in EtOH's actions has been mostly obtained in rats. Studies in mice have mainly focused on $\mathrm{C} 57 \mathrm{BL} / 6 \mathrm{~J}$ and $\mathrm{DBA} / 2 \mathrm{~J}$ mice, two of the most widely used inbred strains in addiction research because of their different sensitivity to several drugs of abuse (Belknap et al., 1993a,b). With respect to alcohol, DBA/2J mice have more severe handling-induced convulsions after acute (Roberts et al., 1992) and chronic (Crabbe et al., 1983) EtOH withdrawal. Further, DBA/2J mice show greater acute functional tolerance (Gallaher et al., 1996), locomotor activity (Crabbe et al., 1983), and hypnosis (Linsenbardt et al., 2009). These strains also differ in EtOH preference and consumption. In the 2-bottle-choice paradigm, C57BL/6J mice drink alcohol, while DBA/2J mice avoid it (Belknap et al., 1977; Phillips et al., 1994). However, when allowed to self-administer EtOH intravenously, these 2 strains do not differ in the amount of EtOH self-administered, suggesting that EtOH's reinforcing properties are similar in both strains (Grahame and Cunningham, 1997). $\mathrm{C} 57 \mathrm{BL} / 6 \mathrm{~J}$ and DBA/2J mice also differ in the behavioral response to neuroactive steroids. $\mathrm{C} 57 \mathrm{BL} / 6 \mathrm{~J}$ mice are more sensitive to the anxiolytic, locomotor stimulant, and anticonvulsant effects of allopregnanolone than DBA/2J mice (Finn et al., 1997b).

Endogenous allopregnanolone may play a role in sensitivity to $\mathrm{EtOH}$ in these strains. Allopregnanolone modulates EtOH intake (Ford et al., 2005, 2007) and reinstates EtOH seeking behavior in C57BL/6 J mice (Finn et al., 2008); it is also thought to modulate $\mathrm{EtOH}$ withdrawal severity across mouse strains (Finn et al., 2004a; Gililland and Finn, 2007). Acute EtOH administration increases whole brain allopregnanolone levels in male DBA/2J (Gabriel et al., 2004) and C57BL/6J mice, although in this strain, the effect did not reach statistical significance (Finn et al., 2004b). Moreover, we recently reported that a single administration of $\mathrm{EtOH}$ ( $2 \mathrm{~g} / \mathrm{kg}$, i.p.) failed to increase plasma allopregnanolone levels in male DBA/2J mice and it actually decreased them in male C57BL/6J mice (Porcu et al., 2010). To further explore these discrepancies and to better understand the effects of $\mathrm{EtOH}$ on brain neurosteroidogenesis in mice, we examined whether acute $\mathrm{EtOH}$ administration alters cerebrocortical and hippocampal levels of allopregnanolone and progesterone in $\mathrm{C} 57 \mathrm{BL} / 6 \mathrm{~J}$ and $\mathrm{DBA} / 2 \mathrm{~J}$ mice. We further describe the effects of acute EtOH administration to these strains on cerebrocortical and hippocampal levels of corticosterone, the main corticosteroid synthesized by the adrenals following HPA axis activation.

\section{MATERIALS AND METHODS}

\section{Animals}

Male C57BL/6J and DBA/2J mice (8 weeks old) were purchased from Charles River (Calco, Italy). They were housed 5 to 6 per cage under an artificial 12-hour-light, 12-hour-dark cycle (light on from 08:00 to 20:00 hours), a constant temperature of $22 \pm 2{ }^{\circ} \mathrm{C}$, and relative humidity of $65 \%$. They had free access to water and standard laboratory food throughout the experimental period. Animal care and handling was in accordance with the European Parliament and the Council Directive 2010/63/EU. Experimental procedures were approved by the local ethics committee; all efforts were made to minimize animal suffering and to reduce the number of animals used.

\section{Drug Administration}

For the dose-response studies, EtOH $(1,2,3$, or $4 \mathrm{~g} / \mathrm{kg}, 20 \% \mathrm{v} / \mathrm{v}$ in saline) or saline was administered by intraperitoneal (i.p.) injection, 60 minutes before euthanasia; for the time-course studies, animals were euthanized 15, 30, 60, and 120 minutes after $\mathrm{EtOH}$ administration $(2 \mathrm{~g} / \mathrm{kg}$, i.p.); saline-treated mice were euthanized 60 minutes later. Morphine hydrochloride $(5,10$, and $30 \mathrm{mg} / \mathrm{kg}$, expressed as free base; S.a.l.a.r.s., Como, Italy) was dissolved in saline and administered i.p. in a volume of $3 \mathrm{ml} / \mathrm{kg}$; mice were euthanized 60 minutes later. Experiments were conducted in the morning from 09:00 to 11:00 hours to avoid circadian fluctuations in neuroactive steroid levels (Corpechot et al., 1997). Mice were not habituated to handling and injection to avoid potential confounds from differences in habituation across strains, which may influence a drug's pharmacological effect (Ryabinin et al., 1999).

\section{Neuroactive Steroid Assay}

Animals were euthanized by decapitation, and the brain areas were rapidly ( $<1$ minute) dissected, frozen on dry ice, and stored at $-80^{\circ} \mathrm{C}$ until steroid extraction. Blood was collected from the trunk into heparin-coated tubes and centrifuged at $900 \times g$ for 15 minutes; plasma was collected and frozen until assayed for steroids. Allopregnanolone, progesterone, and corticosterone were extracted and purified as previously described (Porcu et al., 2004). Briefly, steroids present in cerebrocortical $(\sim 150 \mathrm{mg}$ of tissue in $1 \mathrm{ml}$ of phosphate-buffered saline) or hippocampal ( $\sim 30 \mathrm{mg}$ of tissue in $0.3 \mathrm{ml}$ of phosphate-buffered saline) homogenates were extracted 4 times with an equal volume of ethyl acetate. The combined organic phases were dried under vacuum, and the resulting residue was dissolved in $n$-hexane and applied to Seppak-silica cartridges (Waters, Milan, Italy), and residue components were eluted with a mixture of $n$-hexane:1-propanol $(7: 3, v / v)$. Steroids were further purified by highperformance liquid chromatography (Beckman-Coulter, Cassina de' Pecchi, Italy) on a 5- $\mu \mathrm{m}$ LiChrosorb-diol column $(250 \times 4 \mathrm{~mm}$; Phenomenex, Castel Maggiore, Italy) with a gradient of 1-propanol in $n$-hexane. The recovery of each steroid through the extractionpurification procedures ( 70 to $80 \%$ ) was monitored by adding trace amounts ( $~ 8000 \mathrm{cpm})$ of ${ }^{3} \mathrm{H}$-labeled standards (Perkin Elmer, Mon$\mathrm{za}$, Italy) to the tissue homogenate. Steroid concentrations were quantified by radioimmunoassay. Progesterone (purity $\geq 99 \%$ ), allopregnanolone (purity $\geq 99 \%$ ), and corticosterone (purity $\geq 98.5 \%$ ) were purchased from Sigma-Aldrich (Milan, Italy). Antisera to progesterone and corticosterone were purchased from MP Biomedicals (Solon, $\mathrm{OH}$ ). The progesterone antiserum cross-reacts with 
progesterone $100 \%$, desoxycorticosterone $2.1 \%$, corticosterone $1.4 \%, 17 \alpha$-hydroxypregnenolone $1.4 \%$, pregnenolone $0.2 \%, 20 \alpha-$ dihydroprogesterone $0.2 \%$, testosterone $0.09 \%$, androstenedione $0.07 \%$, and 11 -desoxycortisol $0.05 \%$. The corticosterone antiserum cross-reacts with corticosterone $100 \%$, desoxycorticosterone $2.3 \%$, testosterone $0.47 \%$, prednisolone $0.35 \%, 17 \alpha$-hydroxyprogesterone $0.33 \%$, cortisol $0.27 \%$, progesterone $0.17 \%$, 11-desoxycortisol $0.14 \%, 20 \alpha$-dihydroprogesterone $0.07 \%$ and aldosterone $0.05 \%$. Rabbit antiserum to allopregnanolone was generated and characterized by Dr. R.H. Purdy as previously described (Purdy et al., 1990); it cross-reacts with allopregnanolone $100 \%$, progesterone $17 \%$, and corticosterone $0 \%$ (for a complete cross-reactivity report, see Purdy et al., 1990). All other reagents and organic solvents (high-performance liquid chromatography grade) were of the best available quality from commercial sources. Intra- and interassay variation ranged as follows: progesterone 5 to 8 and 9 to $12 \%$, allopregnanolone 5 to 7 and 8 to $12 \%$, corticosterone 5 to 7 and 9 to $12 \%$, respectively. The minimal detectable limit is $0.01 \mathrm{ng} / \mathrm{g}$ for all steroids.

Progesterone and corticosterone plasma concentrations were assayed by ELISA (Demeditec Diagnostics, Kiel-Wellsee, Germany). Cross-reactivity for the progesterone assay is: progesterone $100 \%$, pregnenolone $5.5 \%, 11$-desoxycorticosterone $1.8 \%, 17 \alpha$-hydroxyprogesterone $0.6 \%$, testosterone $0.14 \%$, and corticosterone $0.3 \%$. Cross-reactivity for the corticosterone assay is: corticosterone $100 \%, 11$-desoxycorticosterone $2.4 \%$, progesterone $0.7 \%$, cortisol $0.3 \%$, and aldosterone $0.2 \%$. Intra- and interassay variation ranges between 5 to 9 and 6 to $8 \%$ for progesterone and 3 to 8 and 5 to $12 \%$ for corticosterone, respectively. The minimal detectable limit is $0.04 \mathrm{ng} / \mathrm{ml}$ for progesterone and $4 \mathrm{ng} / \mathrm{ml}$ for corticosterone.

\section{Statistical Analysis}

Statistical analysis was performed using a commercially available statistical program (GraphPad Prism 5.0; GraphPad Software, San Diego, CA). Data are presented as means \pm SEM, and statistical comparisons were performed by 2-way analysis of variance (ANOVA) followed by Bonferroni post hoc test. A $p$-value $<0.05$ was considered statistically significant.

\section{RESULTS}

Effects of Acute EtOH Administration on Cerebrocortical and Hippocampal Levels of Progesterone and Allopregnanolone in C57BL/6J and DBA/2J Mice

Figure 1 shows the effects of EtOH administration on cerebrocortical concentrations of progesterone and allopregnanolone in $\mathrm{C} 57 \mathrm{BL} / 6 \mathrm{~J}$ and $\mathrm{DBA} / 2 \mathrm{~J}$ mice. None of the EtOH doses examined $(1,2,3$, and $4 \mathrm{~g} / \mathrm{kg})$ significantly affected cerebrocortical levels of progesterone and allopregnanolone in these strains. Two-way ANOVA for progesterone showed no significant effect of EtOH dose, $F(4,150)=0.93, p=0.45$, no significant effect of strain, $F(1$, $150)=1.91, p=0.17$, and no interaction, $F(4,150)=0.13$, $p=0.97$. Two-way ANOVA for allopregnanolone showed no effect of EtOH dose, $F(4,148)=0.14, p=0.97$, no effect
A

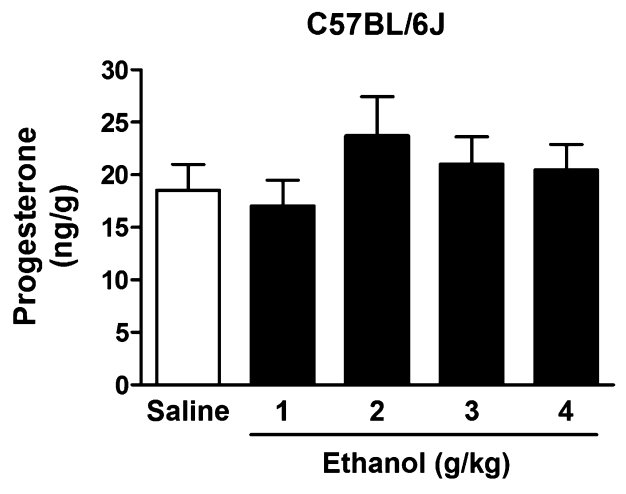

C

\section{C57BL/6J}

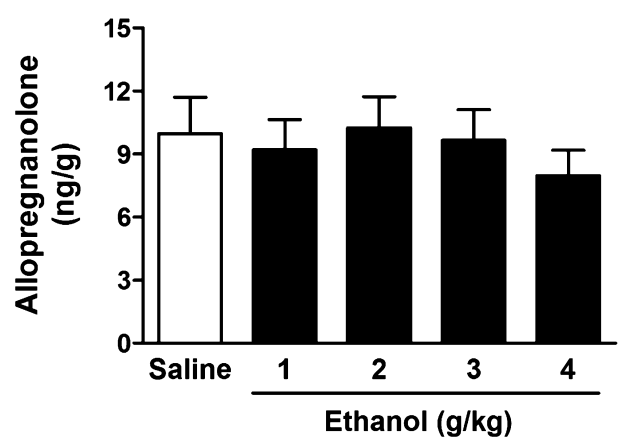

B

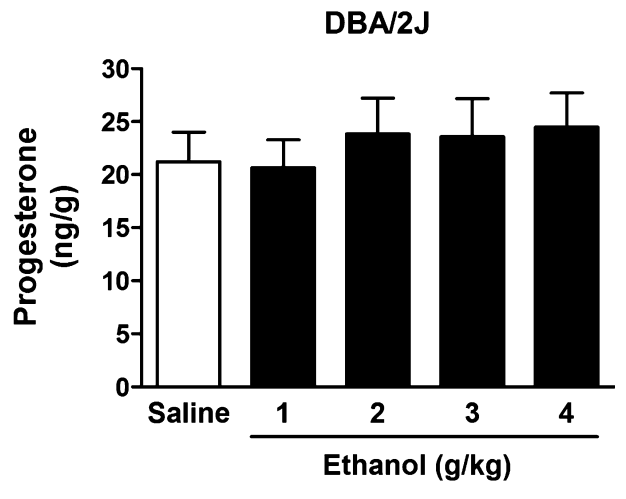

D

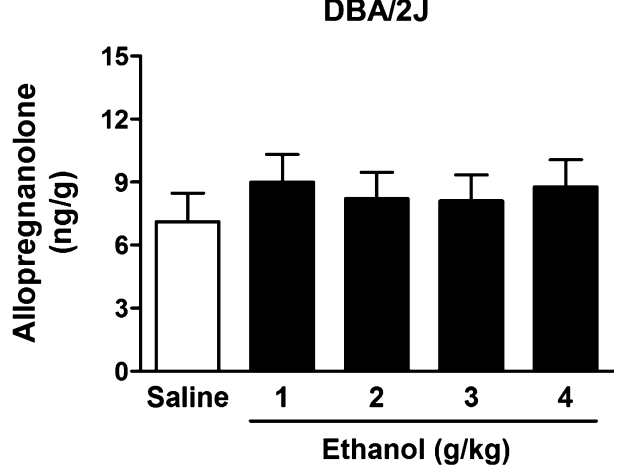

Fig. 1. Effect of ethanol $(\mathrm{EtOH})$ administration on progesterone $(\mathbf{A}, \mathbf{B})$ and allopregnanolone $(\mathbf{C}, \mathbf{D})$ levels in the cerebral cortex of $\mathrm{C} 57 \mathrm{BL} / 6 \mathrm{~J}$ and $\mathrm{DBA} /$ 2J mice. EtOH (1, 2, 3, and $4 \mathrm{~g} / \mathrm{kg}, 20 \% \mathrm{v} / \mathrm{v}$ in saline) or saline was administered i.p. 60 minutes before euthanasia. Levels are expressed as nanograms of steroid per gram of tissue and are average \pm SEM of values from 16 mice per group with the exception of the 3 and $4 \mathrm{~g} / \mathrm{kg}$ groups for allopregnanolone levels in DBA/2J mice where $n=15$. 
of strain, $F(1,148)=1.79, p=0.18$, and no interaction, $F(4,148)=0.55, p=0.69$.

Likewise, acute EtOH administration did not alter hippocampal levels of progesterone and allopregnanolone in C57BL/6J and DBA/2J mice. Two-way ANOVA for progesterone showed no effect of EtOH dose, $F(4,150)=0.94$, $p=0.44$, no effect of strain, $F(1,150)=0.004, p=0.95$, and no interaction, $F(4,150)=0.74, p=0.56$ (Fig. $2 A, B)$. Twoway ANOVA for allopregnanolone showed no effect of EtOH dose, $F(4,148)=1.70, p=0.15$, a significant effect of strain, $F(1,148)=10.63, p=0.001$, and no interaction, $F(4,148)=0.05, p=0.99$. However, the Bonferroni post hoc test failed to identify any significant differences between selected experimental groups within the strains (Fig. 2C,D).

For these experiments, mice were euthanized 60 minutes after EtOH administration, the optimal time point for the EtOH-induced increases in neuroactive steroids in rats (VanDoren et al., 2000). However, EtOH's actions across time may differ between species. Thus, we evaluated the time course for the EtOH-induced changes in neuroactive steroid levels. Acute EtOH administration $(2 \mathrm{~g} / \mathrm{kg}$, i.p.) to $\mathrm{C} 57 \mathrm{BL} / 6 \mathrm{~J}$ and $\mathrm{DBA} / 2 \mathrm{~J}$ mice failed to alter cerebrocortical levels of progesterone and allopregnanolone at any of the time points examined. Two-way ANOVA for progesterone

A

C57BL/6J

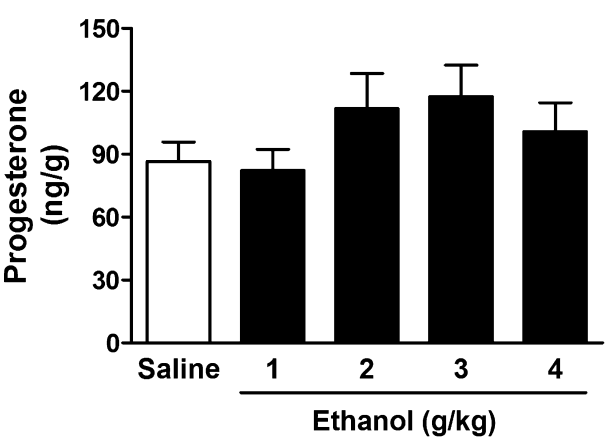

C

C57BL/6J

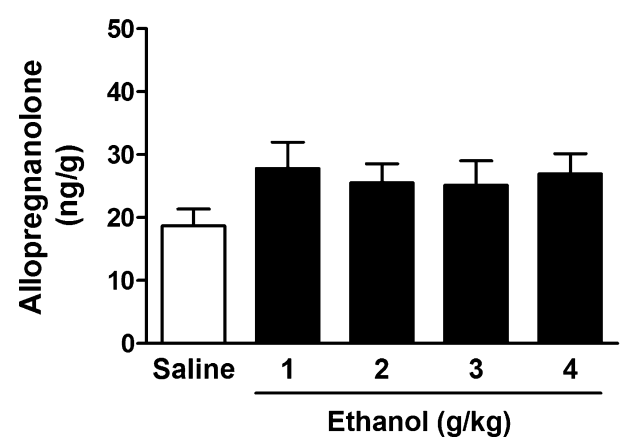

showed no effect of time, $F(4,150)=1.82, p=0.13$, no effect of strain, $F(1,150)=2.39, p=0.12$, and no interaction, $F(4,150)=0.72, p=0.58$ (Fig. 3A, 3B). Two-way ANOVA for allopregnanolone showed no effect of time, $F$ $(4,150)=0.44, p=0.78$, a significant effect of strain, $F(1,150)=18.28, \quad p<0.0001$, but no interaction, $F(4,150)=0.22, p=0.93$. The post hoc test did not identify significant differences between selected groups within the strains (Fig. 3C,D).

Effects of Acute EtOH Administration on Cerebrocortical and Hippocampal Corticosterone Levels in C57BL/6J and DBA/ $2 J$ Mice

To determine whether the lack of EtOH's effects on progesterone and allopregnanolone levels might be due to alterations in HPA axis activation in C57BL/6J and DBA/2 J mice, we measured corticosterone levels in the cerebral cortex and hippocampus. Two-way ANOVA for cerebrocortical corticosterone showed a significant effect of EtOH dose, $F(4,148)=$ $36.78, \quad p<0.0001$, a significant effect of strain, $F(1$, $148)=63.92, p<0.0001$, and a significant interaction, $F(4,148)=4.19, p=0.003$. Acute EtOH administration dose-dependently increased corticosterone levels in the
B



D



Fig. 2. Effect of ethanol $(\mathrm{EtOH})$ administration on progesterone $(\mathbf{A}, \mathbf{B})$ and allopregnanolone $(\mathbf{C}, \mathbf{D})$ levels in the hippocampus of C57BL/6J and DBA/ $2 \mathrm{~J}$ mice. $\mathrm{EtOH}(1,2,3$, and $4 \mathrm{~g} / \mathrm{kg}, 20 \% \mathrm{v} / \mathrm{v}$ in saline) or saline was administered i.p. 60 minutes before euthanasia. Levels are expressed as nanograms of steroid per gram of tissue and are average \pm SEM of values from 16 mice per group with the exception of the 3 and $4 \mathrm{~g} / \mathrm{kg}$ groups for allopregnanolone levels in DBA/2J mice where $n=15$. 
A



C

C57BL/6J

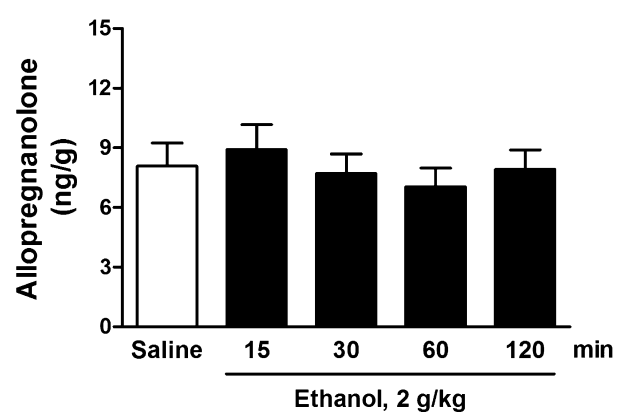

B

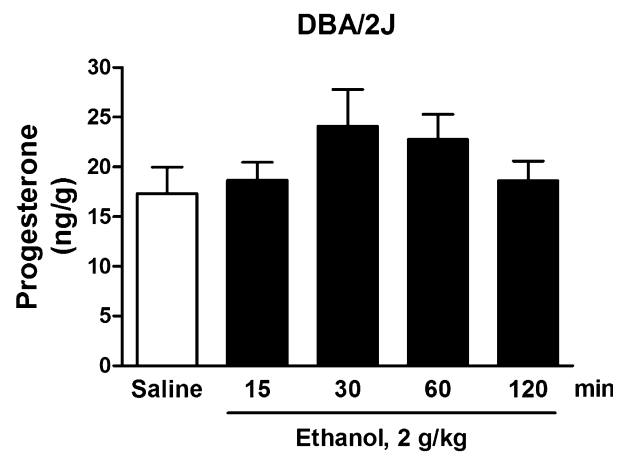

D

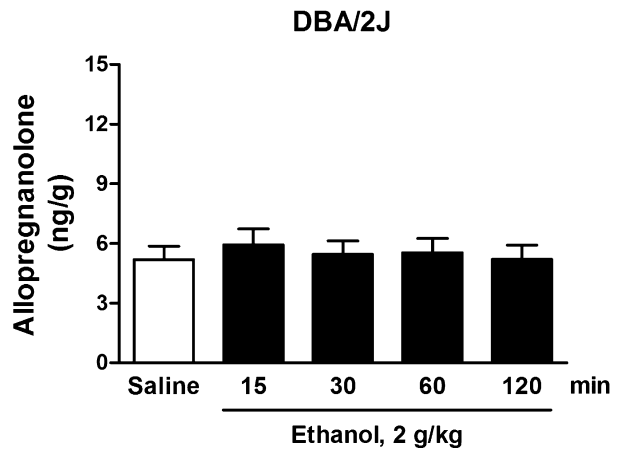

Fig. 3. Time course of the cerebrocortical concentrations of progesterone $(\mathbf{A}, \mathbf{B})$ and allopregnanolone $(\mathbf{C}, \mathbf{D})$ following ethanol $(\mathrm{EtOH})$ administration in C57BL/6J and DBA/2J mice. Mice were injected with $\mathrm{EtOH}(2 \mathrm{~g} / \mathrm{kg}$, i.p.) at the indicated times before euthanasia. Control mice received an equivalent volume of saline and were euthanized 60 minutes later. Levels are expressed as nanograms of steroid per gram of tissue and are average \pm SEM of values from 16 mice per group.

cerebral cortex of C57BL/6J mice $(+319,+347$, and $+459 \%$, at the doses of 2,3 , and $4 \mathrm{~g} / \mathrm{kg}$, respectively, $p<0.001$, Fig. $4 A)$ and DBA $/ 2 \mathrm{~J}$ mice $(+371,+507,+533$, and $+692 \%$, at the doses of $1,2,3$, and $4 \mathrm{~g} / \mathrm{kg}$, respectively, $p<0.001$, Fig. $4 B$ ). The post hoc analysis further revealed a significant difference between strains with respect to $\mathrm{EtOH}$ treatment: The EtOH-induced increase in corticosterone levels was significantly greater $(p<0.01)$ in $\mathrm{DBA} / 2 \mathrm{~J}$ versus $\mathrm{C} 57 \mathrm{BL} / 6 \mathrm{~J}$ mice at all doses examined (Fig. $4 A, B$ ).

Two-way ANOVA for hippocampal corticosterone showed a significant effect of EtOH dose, $F(4,148)=24.31$, $p<0.0001$, no effect of strain, $F(1,148)=2.86, p=0.09$, and no interaction, $F(4,148)=1.09, p=0.36$. Acute EtOH administration dose-dependently increased hippocampal corticosterone levels in C57BL/6J $(+253,+248$, and $+387 \%$, at the doses of 2,3 , and $4 \mathrm{~g} / \mathrm{kg}$, respectively, $p<0.001$, Fig. $4 C)$ and DBA $/ 2 \mathrm{~J}$ mice $(+285 \%$, at $1 \mathrm{~g} / \mathrm{kg}, p<0.01$, and $+441,+554$, and $+661 \%$, at the doses of 2,3 , and $4 \mathrm{~g} / \mathrm{kg}$, respectively, $p<0.001$, Fig. $4 D$ ).

The effects of EtOH on cerebrocortical corticosterone levels were also time dependent. Two-way ANOVA showed a significant effect of time, $F(4,150)=50.66, p<0.0001$, a significant effect of strain, $F(1,150)=4.05, p=0.046$, and a significant interaction, $F(4,150)=8.20, p<0.0001$. In C57BL $/ 6 \mathrm{~J}$ mice, EtOH ( $2 \mathrm{~g} / \mathrm{kg}$, i.p.) increased cerebrocortical corticosterone levels by +214 and $+227 \%$ at 15 and
30 minutes, respectively $(p<0.001)$; corticosterone levels peaked at 60 minutes $(+352 \%, p<0.001)$ and remained elevated at 120 minutes $(+263 \%, p<0.001)$ after $\mathrm{EtOH}$ administration (Fig. 5A). In DBA/2J mice, cerebrocortical corticosterone levels increased at 30 minutes $(+507 \%$, $p<0.001)$, peaked at 60 minutes $(+956 \%, p<0.001)$, and remained elevated at 120 minutes $(+759 \%, p<0.001)$ after EtOH administration (Fig. 5B). The post hoc analysis revealed a significant difference between strains with respect to EtOH treatment: The EtOH-induced increase in corticosterone levels was significantly greater $(p<0.01)$ in DBA/2J versus $\mathrm{C} 57 \mathrm{BL} / 6 \mathrm{~J}$ mice at 60 and 120 minutes; in contrast, at 15 minutes, the effect was greater $(p<0.05)$ in $\mathrm{C} 57 \mathrm{BL} / 6 \mathrm{~J}$ versus DBA/2J mice.

\section{Effects of Acute EtOH Administration on Plasma Levels of Progesterone and Corticosterone in C57BL/6J and DBA/2J Mice}

We further examined EtOH's effects on plasma progesterone and corticosterone levels in C57BL/6J and DBA/2J mice. Two-way ANOVA for progesterone showed a significant effect of time, $F(4,72)=5.25, p=0.0009$, a significant effect of strain, $F(1,72)=36.56, p<0.0001$, but no interaction, $F(4,72)=0.90, p=0.47$ (Table 1). Acute EtOH (2 $\mathrm{g} / \mathrm{kg}$, i.p.) increased plasma progesterone levels in C57BL/6J 


\section{Cerebral Cortex}

A

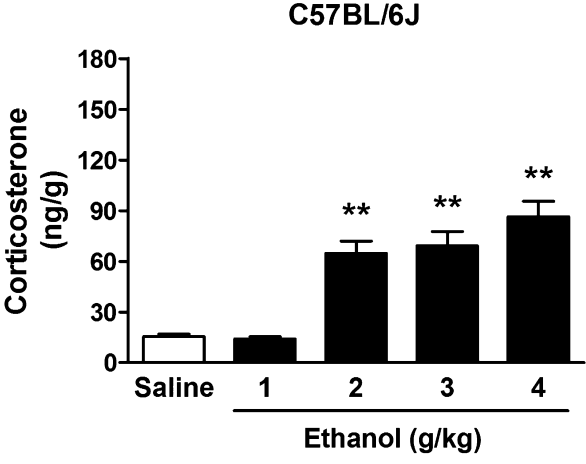

B

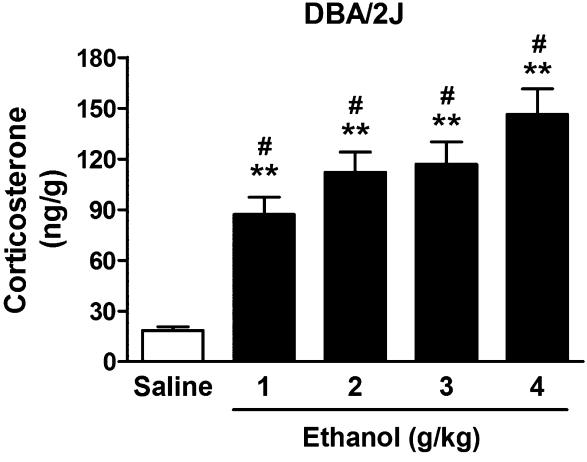

Hippocampus

C

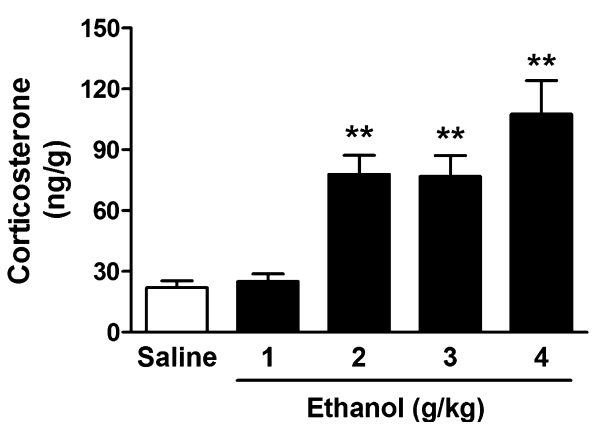

D



Fig. 4. Effect of ethanol (EtOH) administration on corticosterone levels in the cerebral cortex $(\mathbf{A}, \mathbf{B})$ and the hippocampus (C,D) of C57BL/6J and DBA $2 \mathrm{~J}$ mice. $\mathrm{EtOH}(1,2,3$, and $4 \mathrm{~g} / \mathrm{kg}, 20 \% \mathrm{v} / \mathrm{v}$ in saline) or saline was administered i.p. 60 minutes before euthanasia. Levels are expressed as nanograms of steroid per gram of tissue and are average \pm SEM of values from 16 mice per group with the exception of the 3 and $4 \mathrm{~g} / \mathrm{kg}$ groups in DBA $2 \mathrm{~J}$ mice where $n=15$. $* p<0.01$ and $* * p<0.001$ versus the respective saline-treated values; ${ }^{\#} p<0.01$ versus the respective EtOH-treated groups across strains; 2-way ANOVA followed by Bonferroni post hoc test.

A

C57BL/6J

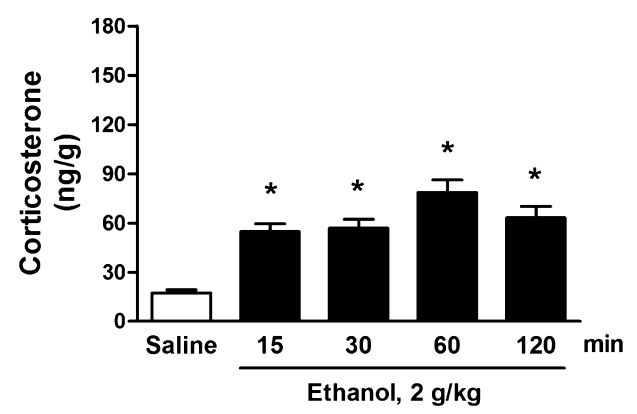

B

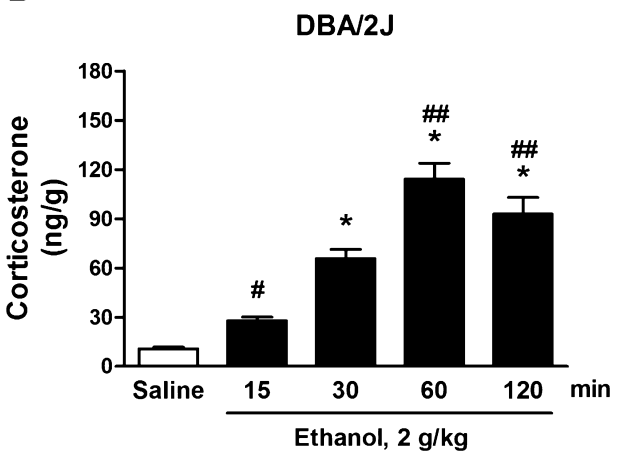

Fig. 5. Time course of the cerebrocortical concentrations of corticosterone following ethanol (EtOH) administration in C57BL/6J (A) and DBA/2J (B) mice. Mice were injected with $\mathrm{EtOH}(2 \mathrm{~g} / \mathrm{kg}$, i.p.) at the indicated times before euthanasia. Control mice received an equivalent volume of saline and were euthanized 60 minutes later. Levels are expressed as nanograms of steroid per gram of tissue and are average \pm SEM of values from 16 mice per group. $* p<0.001$ versus the respective saline-treated values; ${ }^{\#} p<0.05$ and ${ }^{\# \#} p<0.01$ versus the respective EtOH-treated groups across strains; 2-way ANOVA followed by Bonferroni post hoc test.

mice $(+210 \%, p<0.001 ;+173 \%, p<0.01 ;$ and $+230 \%$, $p<0.001$, respectively, at 15,30 , and 60 minutes after administration). Acute EtOH administration increased plasma progesterone levels in DBA/2J mice, although this effect was not statistically significant. The post hoc analysis also revealed a significant difference between strains with respect to the $\mathrm{EtOH}$ treatment groups at 15, 30, and 60 minutes $(p<0.01)$.

Acute EtOH administration increased plasma corticosterone levels in both mouse strains (Table 1). Two-way ANOVA showed a significant effect of time, $F(4$, $75)=96.09, p<0.0001$, no effect of strain, $F(1,75)=0.22$, 
Table 1. Time Course of the Plasma Concentrations of Progesterone and Corticosterone Following Ethanol (EtOH) Administration in C57BL/6J and DBA/2J Mice

\begin{tabular}{|c|c|c|c|c|}
\hline & \multicolumn{2}{|c|}{ Progesterone } & \multicolumn{2}{|c|}{ Corticosterone } \\
\hline & C57BL/6J & DBA/2J & C57BL/6J & DBA/2J \\
\hline Saline & $1.84 \pm 0.54$ & $0.37 \pm 0.08$ & $50.9 \pm 6.97$ & $34.4 \pm 6.95$ \\
\hline $15 \mathrm{~min}$ & $5.71 \pm 0.62^{\star \star}$ & $1.80 \pm 0.42^{\#}$ & $174.3 \pm 8.87^{\star \star}$ & $127.8 \pm 9.35^{\star \star}$ \\
\hline $30 \mathrm{~min}$ & $5.04 \pm 0.79^{\star}$ & $1.77 \pm 0.55^{\#}$ & $194.6 \pm 11.27^{\star \star}$ & $183.5 \pm 9.39^{\star *}$ \\
\hline $60 \mathrm{~min}$ & $6.08 \pm 1.21^{\star \star}$ & $2.74 \pm 0.14^{\#}$ & $248.6 \pm 12.32^{\star \star}$ & $289.3 \pm 14.06^{\star *}$ \\
\hline $120 \mathrm{~min}$ & $3.81 \pm 1.06$ & $1.78 \pm 0.35$ & $174.5 \pm 19.70^{\star \star}$ & $225.7 \pm 13.74^{\star \star *}$ \\
\hline
\end{tabular}

Mice were injected with $\mathrm{EtOH}(2 \mathrm{~g} / \mathrm{kg}$, i.p.) at the indicated times before euthanasia. Control mice received an equivalent volume of saline and were euthanized 60 minutes later. Levels are expressed as nanograms of steroid per milliliter of plasma and are average \pm SEM of values from 9 C57BL/6J mice per group and from $8 \mathrm{DBA} / 2 \mathrm{~J}$ mice per group with the exception of the saline-treated group for progesterone levels where $n=5$.

$* p<0.01$ and $* * p<0.001$ vs the respective saline-treated group, ${ }^{\#} p<0.01$ vs the respective EtOH-treated groups across strains; 2 -way ANOVA followed by Bonferroni post hoc test.

$p=0.64$, and a significant interaction, $F(4,75)=5.88, p=$ 0.0004. In C57BL/6J mice, the effect was apparent at 15 minutes $(+243 \%, p<0.001)$, persisted at 30 minutes $(+282 \%$, $p<0.001)$, peaked at 60 minutes $(+389 \%, p<0.001)$, and remained elevated at 120 minutes $(+243 \%, p<0.001)$. In DBA/2J mice, the effect was apparent at 15 minutes $(+272 \%$, $p<0.001)$, persisted at 30 minutes $(+434 \%, p<0.001)$, peaked at 60 minutes $(+742 \%, p<0.001)$, and remained dramatically elevated at 120 minutes $(+557 \%, p<0.001)$.

\section{Effects of Acute Morphine Administration on Cerebrocortical Levels of Progesterone and Allopregnanolone in C57BL/6J and $D B A / 2 J$ Mice}

Given that acute EtOH failed to alter cerebrocortical and hippocampal levels of progesterone and allopregnanolone, despite a robust HPA axis activation and subsequent increased corticosterone levels, we further evaluated whether the lack of neurosteroidogenesis in C57BL/6J and DBA/2J mice was specific to EtOH. We thus examined the effects of acute morphine administration, which also increases neuroactive steroids in rat cerebral cortex (Concas et al., 2006). Two-way ANOVA for cerebrocortical progesterone levels showed a significant effect of morphine dose, $F(3,70)=$ $10.78, \quad p<0.0001$, a significant effect of strain, $F(1$, $70)=12.08, p=0.0009$, and a significant interaction, $F(3$, $70)=4.61, \quad p=0.005$. Acute morphine administration increased progesterone levels in C57BL/6J $(+117$ and $+107 \%$, respectively, at 10 and $30 \mathrm{mg} / \mathrm{kg}, \quad p<0.001$, Fig. $6 A)$ and DBA/2J mice $(+57$ and $+62 \%$, respectively, at 5 and $10 \mathrm{mg} / \mathrm{kg}, p<0.01$, Fig. $6 B)$. The post hoc analysis revealed a significant difference $(p<0.001)$ between strains at the $5 \mathrm{mg} / \mathrm{kg}$ dose, with the effect being apparent in DBA/ $2 \mathrm{~J}$, but not C57BL/6J mice (Fig. $6 A, B$ ).

Two-way ANOVA for cerebrocortical allopregnanolone levels showed a significant effect of morphine dose, $F(3,72)$ $=12.60, p<0.0001$, a significant effect of strain, $F(1,72)$ $=87.35, \quad p<0.0001$, and a significant interaction, $F(3,72)=3.61, p=0.017$. Acute morphine administration increased allopregnanolone levels in C57BL/6J mice $(+77,+93$ and $+88 \%$, respectively, at 5,10 , and $30 \mathrm{mg} / \mathrm{kg}, p<0.001$,
Fig. $6 C$ ), while in DBA/2J mice levels were increased only at $5 \mathrm{mg} / \mathrm{kg}(+81 \%, p<0.05$, Fig. $6 D)$. The post hoc analysis revealed a significant difference $(p<0.001)$ between strains for all the doses of morphine tested, with an overall greater effect in C57BL/6J versus DBA/2J mice (Fig. $6 C, D$ ).

\section{DISCUSSION}

Several studies have shown that acute EtOH administration increases brain and plasma levels of allopregnanolone and its precursor progesterone in rats (Barbaccia et al., 1999; Morrow et al., 1999; VanDoren et al., 2000) and that EtOHinduced elevations in allopregnanolone concentrations contribute to sensitivity to behavioral and electrophysiological effects of EtOH (Morrow et al., 2006). We recently reported that acute EtOH administration did not alter serum allopregnanolone concentrations in $\mathrm{DBA} / 2 \mathrm{~J}$ mice and it actually decreased them in C57BL/6J mice (Porcu et al., 2010). We further observed that acute $\mathrm{EtOH}$ administration did not alter serum allopregnanolone concentrations in cynomolgus monkeys and humans, suggesting that there might be important species differences in the neurosteroidogenic effects of EtOH (Porcu et al., 2010). Thus, we examined the effects of acute EtOH administration on cerebrocortical and hippocampal neuroactive steroid concentrations in naïve C57BL/ $6 \mathrm{~J}$ and DBA $/ 2 \mathrm{~J}$ male mice, given that these are 2 inbred strains most widely used in alcoholism research, which have been characterized extensively in terms of their behavioral sensitivity to EtOH (Belknap et al., 1993b; Crabbe et al., 1983; Gallaher et al., 1996; Linsenbardt et al., 2009; Roberts et al., 1992). We found that acute $\mathrm{EtOH}$ administration did not alter cerebrocortical and hippocampal concentrations of allopregnanolone and progesterone in naïve $\mathrm{C} 57 \mathrm{BL} / 6 \mathrm{~J}$ and DBA/2J mice at any of the doses and time points examined.

Previous studies on the neurosteroidogenic effects of acute $\mathrm{EtOH}$ in mice have focused on $\mathrm{C} 57 \mathrm{BL} / 6 \mathrm{~J}$ and DBA/2J strains. EtOH administration $(2 \mathrm{~g} / \mathrm{kg})$ increased whole brain allopregnanolone concentrations in $\mathrm{DBA} / 2 \mathrm{~J}$ mice that, 3 weeks before this experiment, underwent an EtOHinduced conditioned place preference test (Gabriel et al., 2004), which may have influenced stress and $\mathrm{EtOH}$ 
A C57BL/6J

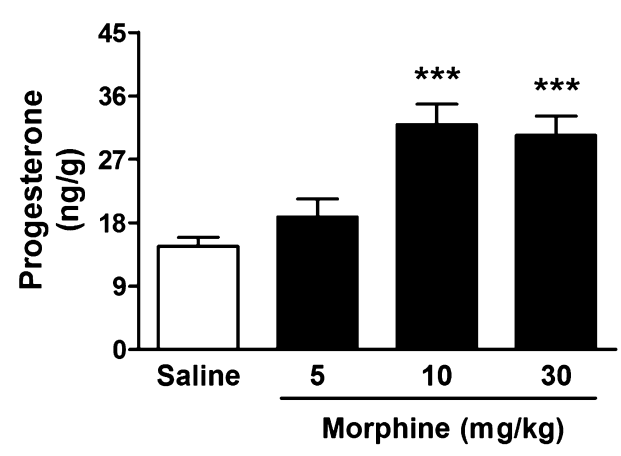

C

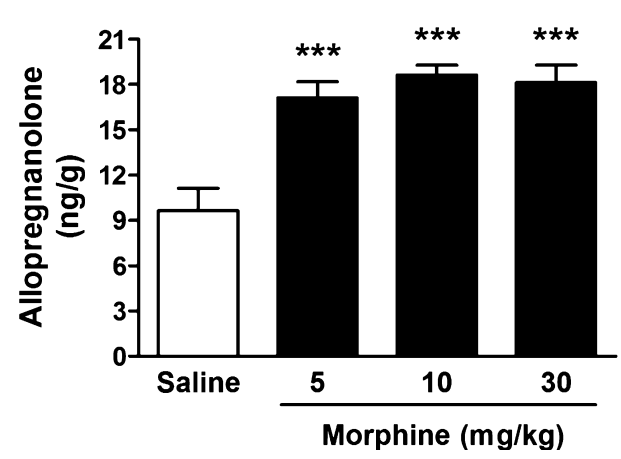

B

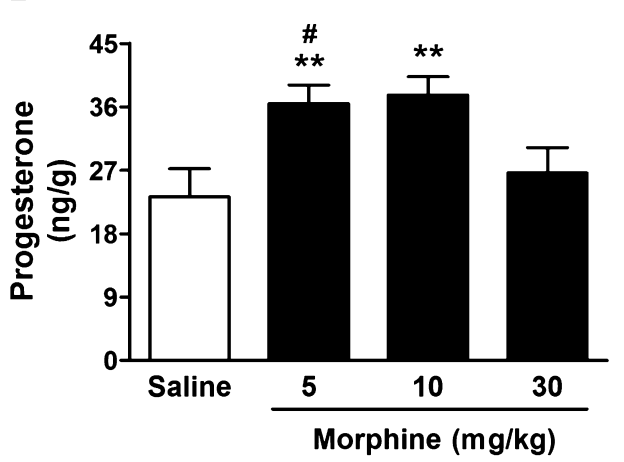

D

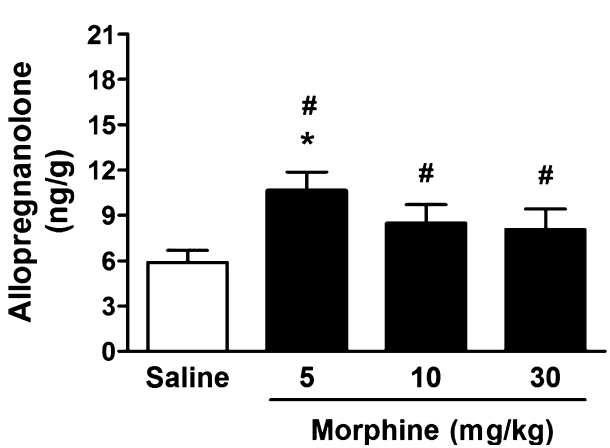

Fig. 6. Effect of morphine administration on progesterone (A,B) and allopregnanolone (C,D) levels in the cerebral cortex of C57BL/6J and DBA/2J mice. Morphine (5, 10, and $30 \mathrm{mg} / \mathrm{kg}$, in saline) or saline was administered i.p. 60 minutes before euthanasia. Levels are expressed as nanograms of steroid per gram of tissue and are average \pm SEM of values from $11 \mathrm{C} 57 \mathrm{BL} / 6 \mathrm{~J}$ mice per group and from $9 \mathrm{DBA} / 2 \mathrm{~J}$ mice per group with the exception of the $5 \mathrm{mg} / \mathrm{kg}$ group for progesterone levels where $n=7 . * p<0.05, * * p<0.01$, and $* * * p<0.001$ versus the respective saline-treated values; ${ }^{\#} p<0.001$ versus the respective morphine-treated groups across strains; 2-way ANOVA followed by Bonferroni post hoc test.

responses. Acute $\mathrm{EtOH}$ administration also increased brain allopregnanolone levels in $\mathrm{C} 57 \mathrm{BL} / 6 \mathrm{~J}$ mice, although this effect did not reach statistical significance, perhaps because data in this experiment were combined from grouped-housed and isolated mice (Finn et al., 2004b). Given that social isolation influences EtOH sensitivity (Serra et al., 2003), we cannot rule out the possibility that isolated $\mathrm{C} 57 \mathrm{BL} / 6 \mathrm{~J}$ mice actually show a neurosteroidogenic response to $\mathrm{EtOH}$, as opposed to group-housed ones, which may have influenced the above mentioned findings. Our experiments were performed in naïve group-housed nonhandled mice, to avoid potential confounds from previous experimental manipulation and from differences in handling habituation across $\mathrm{C} 57 \mathrm{BL} / 6 \mathrm{~J}$ and DBA/2J strains that may influence a drug's pharmacological effect (Ryabinin et al., 1999). Furthermore, methodological differences in measurement of allopregnanolone levels (whole brain vs. selected areas, tissue purification, and antibody used for the radioimmunoassay) might also contribute to the discrepancies between our results and those previously reported. Additionally, in DBA/2J mice, EtOH may promote neurosteroidogenesis in brain regions other than the cerebral cortex and the hippocampus, which might account for the increase in whole brain allopregnanolone content reported by Gabriel and colleagues (2004). Indeed, differential effects of EtOH on immunohistochemical labeling of allopregnanolone content have been recently reported across several rat brain regions (Cook et al., 2013).

We previously reported that acute $\mathrm{EtOH}$ administration did not alter serum allopregnanolone concentrations in $\mathrm{DBA} / 2 \mathrm{~J}$ mice, but it actually decreased them in C57BL/6J mice (Porcu et al., 2010). Serum allopregnanolone concentrations were measured by gas chromatography-mass spectrometry, a highly sensitive and specific method, which detects trace amounts of neuroactive steroids in a small volume of sample like the one that can be obtained from a mouse. Differences in the methodology (gas chromatography-mass spectrometry vs. radioimmunoassay) might contribute to the discrepancy on the EtOH-induced changes in allopregnanolone content in serum versus cerebral cortex and hippocampus. However, these differential effects of EtOH might also be due to the tissue specificity (serum vs. brain). In the present study, we were unable to measure allopregnanolone levels in serum by radioimmunoassay, due to the small amount of sample obtained from a mouse. However, we did measure progesterone levels in plasma using a commercially available kit that requires small amounts of sample. EtOH administration $(2 \mathrm{~g} / \mathrm{kg})$ increased plasma progesterone levels in a time-dependent 
manner, in $\mathrm{C} 57 \mathrm{BL} / 6 \mathrm{~J}$ and DBA $/ 2 \mathrm{~J}$ mice, although the effect on DBA/2J mice did not reach statistical significance. Indeed, the same treatment failed to affect cerebrocortical progesterone levels, suggesting that $\mathrm{EtOH}$ is regulating peripheral, but not central neurosteroidogenesis in naïve C57BL/6J mice.

The doses of EtOH tested in our experiments increase blood alcohol levels in C57BL/6J and DBA/2J mice to values comparable to the ones reported in rats and sufficient to increase neuroactive steroids (Porcu et al., 2010). EtOH metabolism is faster in mice than in rats (Abel, 1982); however, EtOH fails to alter cerebrocortical and hippocampal allopregnanolone levels in C57BL/6J and DBA/2J mice even at early time points, suggesting that the neurosteroidogenic properties of EtOH in the cerebral cortex and the hippocampus may be species specific.

The lack of effects of EtOH on allopregnanolone levels in naïve $\mathrm{C} 57 \mathrm{BL} / 6 \mathrm{~J}$ and $\mathrm{DBA} / 2 \mathrm{~J}$ mice suggests that alterations in endogenous allopregnanolone concentrations, in contrast to what happens in rats (Morrow et al., 2006), do not appear to modulate EtOH's behavioral effects in these strains, and therefore, endogenous allopregnanolone might not account for the differences in EtOH sensitivity observed in C57BL/6 J versus DBA/2J mice. However, previous studies have suggested that allopregnanolone may indeed play a role in EtOH's actions, independent from its steroidogenic effects. In fact, allopregnanolone administration to $\mathrm{C} 57 \mathrm{BL} / 6 \mathrm{~J}$ mice modulates EtOH self-administration (Ford et al., 2005, 2007) and reinstates EtOH seeking behavior (Finn et al., 2008). Likewise, allopregnanolone administration modulates EtOH self-administration in rats (Janak et al., 1998). Furthermore, neuroactive steroids modulate EtOH withdrawal severity in several mouse strains (Finn et al., 2004a; Gililland and Finn, 2007). However, allopregnanolone, despite having reinforcing properties in DBA/2J mice (Finn et al., 1997a), does not alter EtOH conditioned place preference in this strain (Gabriel et al., 2004).

The EtOH-induced increase in allopregnanolone content contributes to some behavioral effects of EtOH in rats, including anticonvulsant, sedative, and anxiolytic actions, as well as learning and memory (Morrow et al., 2006). Acute $\mathrm{EtOH}$ administration induces similar effects in mice, but fails to alter allopregnanolone levels, suggesting that different mechanisms could mediate similar effects in rats and mice. EtOH has pleiotropic actions on several neuromodulators and neurotransmitters; we can hypothesize that the EtOH-induced increase in allopregnanolone content in rats may contribute to $\mathrm{EtOH}$ sensitivity to a greater extent than other neuromodulators; in contrast, other systems may be more prominent in mice. For instance, the EtOH metabolite acetaldehyde also influences EtOH's effects. Concentrations of acetaldehyde such as those induced by EtOH administration ( $2 \mathrm{~g} / \mathrm{kg}$, i.p.) did not increase allopregnanolone levels in rats (Boyd et al., 2008). However, acetaldehyde has been found to mediate behavioral effects of $\mathrm{EtOH}$ in rats as well as $\mathrm{C} 57 \mathrm{BL} / 6 \mathrm{~J}$ mice (Quertemont et al., 2004), suggesting that its effects may be independent of EtOH-induced neurosteroidogenesis. We cannot rule out the possibility that the EtOH-induced increase in allopregnanolone and the concomitant presence of acetaldehyde may both participate in the sensitivity to EtOH's behavioral effects. Allopregnanolone may have a more prominent role in rats, while acetaldehyde may have more significance in mice. Future studies may address these hypotheses.

In $\mathrm{C} 57 \mathrm{BL} / 6 \mathrm{~J}$ mice, neurosteroids are required to activate the HPA axis in response to stress, whereby they promote a collapse of the chloride gradient resulting in excitatory GABAergic transmission at the level of the corticotrophin-releasing hormone neurons in the hypothalamus (Sarkar et al., 2011). The failure of acute EtOH to increase cerebrocortical and hippocampal allopregnanolone levels may suggest an impairment in EtOH-induced HPA axis activation in C57BL/6J and DBA/2J mice. To evaluate EtOH's activation of the HPA axis in naive C57BL/6J and DBA/2J mice, we measured corticosterone levels, and we found that acute EtOH administration, dose- and time-dependently, increased corticosterone levels in the cerebral cortex, hippocampus, and plasma, suggesting that $\mathrm{EtOH}$ administration is activating the HPA axis. This is the first evidence of brain corticosterone alterations in these 2 mouse strains. This result is in agreement with a previous report showing an increase in brain corticosterone concentrations following acute $\mathrm{EtOH}$ administration in male Tuck-Ordinary (TO) mice (Croft et al., 2008). Hippocampal corticosterone levels were also increased in TO mice following long-term EtOH withdrawal (Little et al., 2008). Overall, these results suggest that EtOH stimulates the HPA axis and promotes corticosterone synthesis not only in the periphery, as was previously shown (Roberts et al., 1992), but also in the cerebral cortex and the hippocampus of both mouse strains.

Corticosterone is the main steroid produced by adrenal glands in response to HPA axis activation and is a progesterone metabolite. Thus, it is quite unusual that EtOH induced an increase in corticosterone levels in brain without altering levels of its precursor. The mechanism by which $\mathrm{EtOH}$ is exerting this effect is still unclear. Perhaps EtOH may selectively alter cerebrocortical and hippocampal steroidogenesis in a manner that produces corticosterone, rather than progesterone from pregnenolone. However, plasma progesterone levels were increased following acute $\mathrm{EtOH}$ administration in C57BL/6J mice. At present, we cannot rule out the possibility that acute EtOH administration may have differential effects on steroidogenic enzymes in the adrenals and brain of these mouse strains.

The lack of effects of EtOH on cerebrocortical allopregnanolone and progesterone concentrations in naïve C57BL/ $6 \mathrm{~J}$ and DBA/2J mice appears to be specific to $\mathrm{EtOH}$, given that administration of morphine increases cerebrocortical allopregnanolone levels in these strains. Morphine increased cerebrocortical concentrations of allopregnanolone and progesterone in male Sprague-Dawley rats, starting at the dose of $10 \mathrm{mg} / \mathrm{kg}$ (Concas et al., 2006). Likewise, in C57BL/6J 
mice, morphine significantly increased cerebrocortical levels of progesterone, in a manner similar to that reported in rats. Cerebrocortical allopregnanolone levels in C57BL/6J mice increased starting at the dose of $5 \mathrm{mg} / \mathrm{kg}$, suggesting that this strain might be even more sensitive than Sprague-Dawley rats to the neurosteroidogenic effects of morphine, although morphine increased allopregnanolone levels to a slightly greater extent in rats than $\mathrm{C} 57 \mathrm{BL} / 6 \mathrm{~J}$ mice. Morphine administration also increased progesterone and allopregnanolone levels in the cerebral cortex of DBA/2J mice, although the effect was less pronounced compared with the one observed in $\mathrm{C} 57 \mathrm{BL} / 6 \mathrm{~J}$ mice, suggesting that these strains have a different sensitivity to the morphine-induced increases in cerebrocortical allopregnanolone levels.

In conclusion, these results demonstrate that acute EtOH administration increases corticosterone levels but does not alter allopregnanolone and progesterone concentrations in the cerebral cortex and the hippocampus of naive C57BL/6J and DBA $/ 2 \mathrm{~J}$ mice, in contrast to what has been observed in rats or in whole brain of $\mathrm{DBA} / 2 \mathrm{~J}$ mice. Our observations may suggest important species differences in the effects of $\mathrm{EtOH}$ on brain neurosteroidogenesis. Furthermore, given that acute $\mathrm{EtOH}$ administration does not increase cerebrocortical and hippocampal allopregnanolone levels in C57BL/ $6 \mathrm{~J}$ and $\mathrm{DBA} / 2 \mathrm{~J}$ mice, the different sensitivity that these strains have to acute EtOH's behavioral effects might not be directly related to neuroactive steroid responses. Finally, the lack of effects of EtOH on cerebrocortical neurosteroidogenesis in $\mathrm{C} 57 \mathrm{BL} / 6 \mathrm{~J}$ and $\mathrm{DBA} / 2 \mathrm{~J}$ mice appears to be an effect selective for $\mathrm{EtOH}$, given that morphine administration increases cerebrocortical allopregnanolone levels in these strains.

\section{ACKNOWLEDGMENTS}

This study was supported by a Sardinian Region Award L.R. n.3/2008 (Interventi per favorire il rientro di docenti e ricercatori in Sardegna) to PP, and by NIH AA020935 and the Bowles Center for Alcohol Studies (Chapel Hill, NC) to ALM.

\section{REFERENCES}

Abel EL (1982) Behavioral teratology of alcohol (animal model studies of the fetal alcohol syndrome), in Fetal Alcohol Syndrome, Vol. III. Animal Studies (Abel EL ed), pp 59-81. CRC Press, Boca Raton, FL.

Barbaccia ML, Affricano D, Trabucchi M, Purdy RH, Colombo G, Agabio R, Gessa GL (1999) Ethanol markedly increases "GABAergic" neurosteroids in alcohol-preferring rats. Eur J Pharmacol 384:R1-R2.

Belknap JK, Belknap ND, Berg JH, Coleman R (1977) Preabsorptive vs. postabsorptive control of ethanol intake in $\mathrm{C} 57 \mathrm{BL} / 6 \mathrm{~J}$ and DBA/2J mice. Behav Genet 7:413-425.

Belknap JK, Crabbe JC, Riggan J, O’Toole LA (1993a) Voluntary consumption of morphine in 15 inbred mouse strains. Psychopharmacology 112:352-358.

Belknap JK, Crabbe JC, Young ER (1993b) Voluntary consumption of ethanol in 15 inbred mouse strains. Psychopharmacology 112:503-510.
Biggio G, Purdy RH (2001) Neurosteroids and Brain Function, International Review of Neurobiology, Vol. 46. Academic Press, New York, NY.

Boyd KN, Kumar S, O’Buckley TK, Porcu P, Morrow AL (2010) Ethanol induction of steroidogenesis in rat adrenal and brain is dependent upon pituitary ACTH release and de novo adrenal StAR synthesis. J Neurochem 112:784-796.

Boyd KN, O'Buckley TK, Morrow AL (2008) Role of acetaldehyde in ethanol-induced elevation of the neuroactive steroid $3 \alpha$-hydroxy-5 $\alpha$-pregnan20-one in rats. Alcohol Clin Exp Res 32:1774-1781.

Concas A, Porcu P, Sogliano C, Serra M, Purdy RH, Biggio G (2000) Caffeine-induced increases in the brain and plasma concentrations of neuroactive steroids in the rat. Pharmacol Biochem Behav 66:39-45.

Concas A, Sogliano C, Porcu P, Marra C, Brundu A, Biggio G (2006) Neurosteroids in nicotine and morphine dependence. Psychopharmacology 186:281-292.

Cook JB, Dumitru AM, O'Buckley TK, Morrow AL (2013) Ethanol administration produces divergent changes in GABAergic neuroactive steroid immunohistochemistry in the rat brain. Alcohol Clin Exp Res doi: 10. 1111/acer.12223 [Epub ahead of print].

Corpechot C, Collins BE, Carey MP, Tsouros A, Robel P, Fry JP (1997) Brain neurosteroids during the mouse oestrous cycle. Brain Res 766: 276-280.

Crabbe JC, Kosobud A, Young ER (1983) Genetic selection for ethanol withdrawal severity: differences in replicate mouse lines. Life Sci 33:955-962.

Croft AP, O'Callaghan MJ, Shaw SG, Connolly G, Jacquot C, Little HJ (2008) Effects of minor laboratory procedures, adrenalectomy, social defeat or acute alcohol on regional brain concentrations of corticosterone. Brain Res 1238:12-22.

Finn DA, Ford MM, Wiren KM, Roselli CE, Crabbe JC (2004a) The role of pregnane neurosteroids in ethanol withdrawal: behavioral genetic approaches. Pharmacol Ther 101:91-112.

Finn DA, Mark GP, Fretwell AM, Gililland-Kaufman KR, Strong MN, Ford MM (2008) Reinstatement of ethanol and sucrose seeking by the neurosteroid allopregnanolone in C57BL/6 mice. Psychopharmacology 201:423-433

Finn DA, Phillips TJ, Okorn DM, Chester JA, Cunningham CL (1997a) Rewarding effect of the neuroactive steroid 3alpha-hydroxy-5alpha-pregnan-20-one in mice. Pharmacol Biochem Behav 56:261-264.

Finn DA, Roberts AJ, Lotrich F, Gallaher EJ (1997b) Genetic differences in behavioral sensitivity to a neuroactive steroid. J Pharmacol Exp Ther 280:820-828.

Finn DA, Sinnott RS, Ford MM, Long SL, Tanchuck MA, Phillips TJ (2004b) Sex differences in the effect of ethanol injection and consumption on brain allopregnanolone levels in C57BL/6 mice. Neuroscience 123:813819

Fish EW, Faccidomo S, DeBold JF, Miczek KA (2001) Alcohol, allopregnanolone and aggression in mice. Psychopharmacology 153:473-483.

Follesa P, Biggio F, Talani G, Murru L, Serra M, Sanna E, Biggio G (2006) Neurosteroids, $\mathrm{GABA}_{\mathrm{A}}$ receptors, and ethanol dependence. Psychopharmacology 186:267-280.

Ford MM, Mark GP, Nickel JD, Phillips TJ, Finn DA (2007) Allopregnanolone influences the consummatory processes that govern ethanol drinking in C57BL/6J mice. Behav Brain Res 179:265-272.

Ford MM, Nickel JD, Phillips TJ, Finn DA (2005) Neurosteroid modulators of $\mathrm{GABA}_{\mathrm{A}}$ receptors differentially modulate ethanol intake patterns in male C57BL/6J mice. Alcohol Clin Exp Res 29:1630-1640.

Gabriel KI, Cunningham CL, Finn DA (2004) Allopregnanolone does not influence ethanol-induced conditioned place preference in $\mathrm{DBA} / 2 \mathrm{~J}$ mice. Psychopharmacology 176:50-56.

Gallaher EJ, Jones GE, Belknap JK, Crabbe JC (1996) Identification of genetic markers for initial sensitivity and rapid tolerance to ethanolinduced ataxia using quantitative trait locus analysis in BXD recombinant inbred mice. J Pharmacol Exp Ther 277:604-612.

Gililland KR, Finn DA (2007) The impact of gonadectomy and adrenalectomy on acute withdrawal severity in male and female C57BL/6J and 
DBA/2J mice following a single high dose of ethanol. Alcohol Clin Exp Res 31:1846-1857.

Grahame NJ, Cunningham CL (1997) Intravenous ethanol self-administration in C57BL/6J and DBA/2J mice. Alcohol Clin Exp Res 21:56-62.

Grobin AC, VanDoren MJ, Porrino LJ, Morrow AL (2005) Cortical 3 $\alpha$ hydroxy-5 $\alpha$-pregnan-20-one levels after acute administration of $\Delta 9$ tetrahydrocannabinol, cocaine and morphine. Psychopharmacology 179:544-550.

Hirani K, Khisti RT, Chopde CT (2002) Behavioral action of ethanol in Porsolt's forced swim test: modulation by 3alpha-hydroxy-5alpha-pregnan-20-one. Neuropharmacology 43:1339-1350.

Hirani K, Sharma AN, Jain NS, Ugale RR, Chopde CT (2005) Evaluation of GABAergic neuroactive steroid 3alpha-hydroxy-5alpha-pregnane-20one as a neurobiological substrate for the anti-anxiety effect of ethanol in rats. Psychopharmacology 180:267-278.

Janak PH, Redfern JE, Samson HH (1998) The reinforcing effects of ethanol are altered by the endogenous neurosteroid, allopregnanolone. Alcohol Clin Exp Res 22:1106-1112.

Khisti RT, VanDoren MJ, O'Buckley T, Morrow AL (2003) Neuroactive steroid 3alpha-hydroxy-5alpha-pregnan-20-one modulates ethanolinduced loss of righting reflex in rats. Brain Res 980:255-265.

Linsenbardt DN, Moore EM, Gross CD, Goldfarb KJ, Blackman LC, Boehm SL 2nd (2009) Sensitivity and tolerance to the hypnotic and ataxic effects of ethanol in adolescent and adult $\mathrm{C} 57 \mathrm{BL} / 6 \mathrm{~J}$ and $\mathrm{DBA} / 2 \mathrm{~J}$ mice. Alcohol Clin Exp Res 33:464 476.

Little HJ, Croft AP, O'Callaghan MJ, Brooks SP, Wang G, Shaw SG (2008) Selective increases in regional brain glucocorticoid: a novel effect of chronic alcohol. Neuroscience 156:1017-1027.

Matthews DB, Morrow AL, Tokunaga S, McDaniel JR (2002) Acute ethanol administration and acute allopregnanolone administration impair spatial memory in the Morris water task. Alcohol Clin Exp Res 26: $1747-1751$.

Morrow AL, Janis GC, VanDoren MJ, Matthews DB, Samson HH, Janak PH, Grant KA (1999) Neurosteroids mediate pharmacological effects of ethanol: a new mechanism of ethanol action? Alcohol Clin Exp Res 23:1933-1940.

Morrow AL, Porcu P, Boyd KN, Grant KA (2006) Hypothalamic-pituitary-adrenal axis modulation of GABAergic neuroactive steroids influences ethanol sensitivity and drinking behavior. Dialogues Clin Neurosci 8:463-477.

O’Dell LE, Alomary AA, Vallee M, Koob GF, Fitzgerald RL, Purdy RH (2004) Ethanol-induced increases in neuroactive steroids in the rat brain and plasma are absent in adrenalectomized and gonadectomized rats. Eur J Pharmacol 484:241-247.
Phillips TJ, Crabbe JC, Metten P, Belknap JK (1994) Localization of genes affecting alcohol drinking in mice. Alcohol Clin Exp Res 18:931-941.

Porcu P, O'Buckley TK, Alward SE, Song SC, Grant KA, de Wit H, Morrow AL (2010) Differential effects of ethanol on serum GABAergic $3 \alpha, 5 \alpha /$ $3 \alpha, 5 \beta$ neuroactive steroids in mice, rats, cynomolgus monkeys, and humans. Alcohol Clin Exp Res 34:432-442.

Porcu P, Sogliano C, Cinus M, Purdy RH, Biggio G, Concas A (2003) Nicotine-induced changes in cerebrocortical neuroactive steroids and plasma corticosterone concentrations in the rat. Pharmacol Biochem Behav 74:683-690.

Porcu P, Sogliano C, Ibba C, Piredda M, Tocco S, Marra C, Purdy RH, Biggio G, Concas A (2004) Failure of gamma-hydroxybutyric acid both to increase neuroactive steroid concentrations in adrenalectomized-orchiectomized rats and to induce tolerance to its steroidogenic effect in intact animals. Brain Res 1012:160-168.

Purdy RH, Morrow AL, Blinn JR, Paul SM (1990) Synthesis, metabolism, and pharmacological activity of $3 \alpha$-hydroxy steroids which potentiate GABA-receptor-mediated chloride ion uptake in rat cerebral cortical synaptoneurosomes. J Med Chem 33:1572-1581.

Quertemont E, Tambour S, Bernaerts P, Zimatkin SM, Tirelli E (2004) Behavioral characterization of acetaldehyde in $\mathrm{C} 57 \mathrm{BL} / 6 \mathrm{~J}$ mice: locomotor, hypnotic, anxiolytic and amnesic effects. Psychopharmacology 177:84-92.

Roberts AJ, Crabbe JC, Keith LD (1992) Genetic differences in hypothalamic-pituitary-adrenal axis responsiveness to acute ethanol and acute ethanol withdrawal. Brain Res 579:296-302.

Ryabinin AE, Wang YM, Finn DA (1999) Different levels of Fos immunoreactivity after repeated handling and injection stress in two inbred strains of mice. Pharmacol Biochem Behav 63:143-151.

Sarkar J, Wakefield S, MacKenzie G, Moss SJ, Maguire J (2011) Neurosteroidogenesis is required for the physiological response to stress: role of neurosteroid-sensitive $\mathrm{GABA}_{\mathrm{A}}$ receptors. J Neurosci 31:1819818210.

Serra M, Pisu MG, Floris I, Cara V, Purdy RH, Biggio G (2003) Social isolation-induced increase in the sensitivity of rats to the steroidogenic effect of ethanol. J Neurochem 85:257-263.

Tokunaga S, McDaniel JR, Morrow AL, Matthews DB (2003) Effect of acute ethanol administration and acute allopregnanolone administration on spontaneous hippocampal pyramidal cell neural activity. Brain Res 967:273-280.

VanDoren MJ, Matthews DB, Janis GC, Grobin AC, Devaud LL, Morrow AL (2000) Neuroactive steroid 3alpha-hydroxy-5alpha-pregnan-20-one modulates electrophysiological and behavioral actions of ethanol. J Neurosci 20:1982-1989. 